\title{
Early detection of disseminated tuberculosis after cord blood transplantation: role of pre-transplant positron emission tomography
}

\author{
Ryohei Abe ${ }^{1}$, Takehiko Mori ${ }^{1,2}$, Masatoshi Sakurai ${ }^{1}$, Jun Kato ${ }^{1}$, Norisato Hashimoto ${ }^{1}$, Taku Kikuchi ${ }^{1}$, Takaaki Toyama ${ }^{1}$, \\ Yuya Koda', Naoki Hasegawa' ${ }^{2}$, Shinichiro Okamoto ${ }^{1}$
}

${ }^{1}$ Division of Hematology, Department of Medicine, Keio University School of Medicine, Tokyo, Japan

${ }^{2}$ Center for Infectious Disease and Infection Control, Keio University Hospital, Tokyo, Japan.

18F-fluorodeoxyglucose-positron emission tomography (PET) is a useful asset for evaluating the disease stage, residual disease, and activity of malignant lymphoma, including adult T-cell leukemia/lymphoma (ATLL), and its use to treat infectious diseases has also been reported. After a 51-year-old female with ATLL received chemotherapy, all of her lesions became undetectable by computed tomography (CT). However, intrathoracic lymph nodes were found to be PET-avid without significant swelling, and were considered recurrent ATLL lesions. She subsequently underwent cord blood transplantation (CBT). Two months later, she presented with high-grade fever. CT revealed significant left hilar and mediastinal lymph node swelling with central low density, suggesting necrosis. After Mycobacterium tuberculosis (M. tuberculosis) was repeatedly detected in her blood, urine, feces, and gastric juice, disseminated tuberculosis was diagnosed. Physicians should always be aware of infectious diseases, particularly tuberculosis, as a possible cause of PET-avid nodal lesions in immunocompromised patients. (Journal of Hematopoietic Cell Transplantation 5 (4): 155-157, 2016.)

\section{Introduction}

18F-fluorodeoxyglucose (FDG) -positron emission tomography $(\mathrm{PET})$ is a useful asset not only for assessing the disease status of hematological malignancies such as malignant lymphomas, but also for detecting infectious lesions. ${ }^{1,2}$ Recently, PET was reported to have higher sensitivity than computed tomography (CT) for detecting organs affected by tuberculosis. ${ }^{3}$ We here report a case of adult T-cell leukemia/lymphoma (ATLL) in which significant uptakes in the intrathoracic lymph nodes by pre-transplant FDG-PET progressed to the disseminated tuberculosis shortly after cord blood transplantation (CBT).

\section{Case report}

A 51-year-old female was diagnosed with lymphoma-type ATLL, which involved the cervical, supraclavicular, axillar, para-aortic, mesenteric, iliac, and inguinal lymph nodes. FDG-PET was not performed at diagnosis. Her medical history was not remarkable for infectious diseases, including tuberculosis. She was initially treated with chemotherapy (VCAP-AMP-VECP; vincristine, cyclophosphamide, doxorubicin, and prednisone-doxorubicin, ranimustine, and prednisone-vindesine, etoposide, carboplatin, and prednisone) and achieved a complete response, as confirmed by CT. She was then referred to our hospital to receive CBT as a curative therapy. However, pre-transplant FDG-PET revealed significant uptakes at the left sub-aortic and hilar lymph nodes (maximum standardized uptake values (SUVs): 7.87 and 7.02, respectively (Figure 1a, b)) without a radiologically significant lymphadenopathy or lung shadows. The lesions were considered to be a recurrence of ATLL, and further examinations, such as histological examinations and interferon-gamma releasing assay, were not performed. CBT from an unrelated donor was promptly performed after being con-

Submitted March 1, 2016; Accepted April 27, 2016. (Handling Editor: Yoshinobu Maeda, Okayama University Hospital)

Key words: positron emission tomography, tuberculosis, lymph node, cord blood transplantation, adult T-cell leukemia/lymphoma

Correspondence: Takehiko Mori, Division of Hematology, Department of Medicine, Keio University School of Medicine, 35 Shinanomachi, Shinjuku-ku, Tokyo 160-8582, Japan. E-mail: tmori@a3.keio.jp

dx.doi.org/10.7889/hct.5.155 (C) The Japan Society for Hematopoietic Cell Transplantation. 


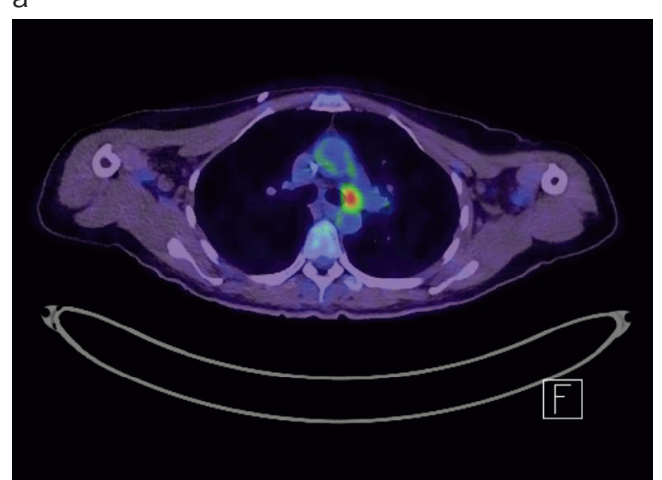

b

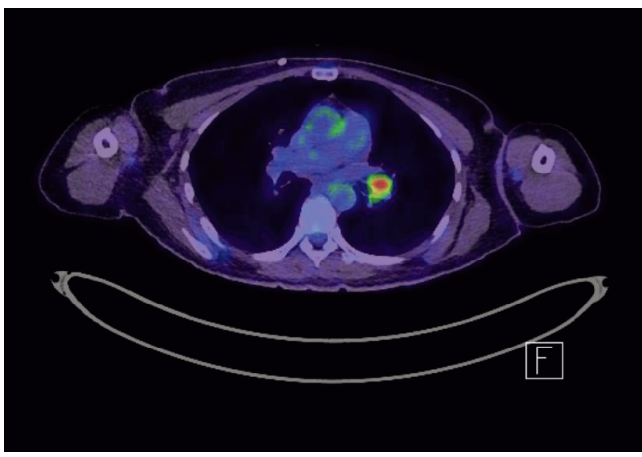

Figure 1. 18F-fluorodeoxy glucose-positron emission tomography (FDP-PET) images before cord blood transplantation. Without significant swelling, significant uptake was demonstrated at the left sub-aortic (a) and hilar (b) lymph nodes. The maximum standardized uptake values were 7.87 and 7.02 , respectively.

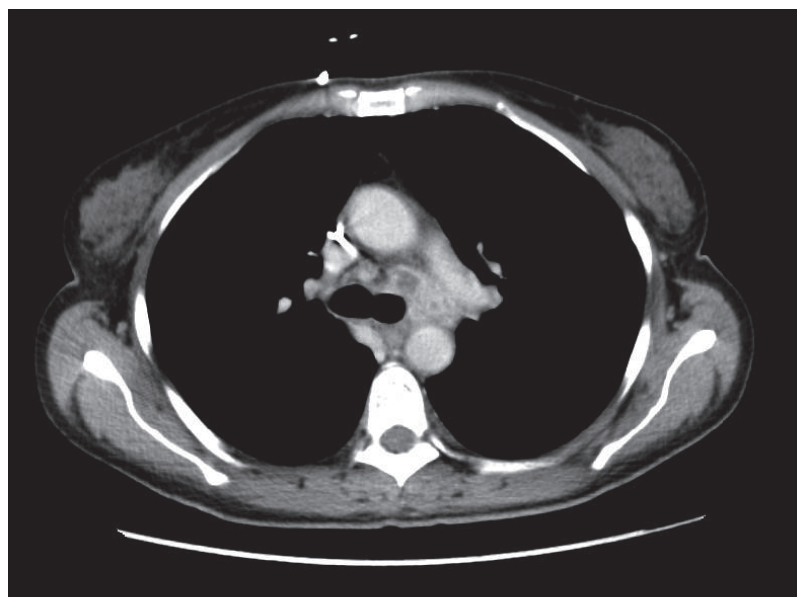

Figure 2. Computed tomography (CT) image 2 months after transplantation. CT revealed significant swelling of the hilar and the mediastinal lymph nodes with a central low density.

ditioned with fludarabine $\left(125 \mathrm{mg} / \mathrm{m}^{2}\right)$, melphalan (140 mg/ $\mathrm{m}^{2}$ ), and total body irradiation (4 Gy). Cyclosporine A and methotrexate were given as a graft-versus-host disease prophylaxis. Prophylactic ciprofloxacin, fluconazole, and acyclovir were given. On day 6 post-transplantation, she developed febrile neutropenia, and ciprofloxacin was switched to tazobactam/piperacillin. The antimicrobial agent was serially switched to meropenem and finally to levofloxacin (500 mg/ day), which was given from day 19 to day 24. Neutrophil engraftment was achieved on day 26 with a complete donor chimerism. On day 29, acute graft-versus-host disease developed along with fever and was successfully treated with prednisolone at a dose of $0.5 \mathrm{mg} / \mathrm{kg}$. Two months after the transplantation, she presented with high-grade fever. CT revealed significant swelling of the left hilar and swelling of the mediastinal lymph nodes with a central low density, suggesting necrosis (Figure 2). After Mycobacterium tuberculosis ( $M$. tuberculosis) was repeatedly detected in the blood, urine, feces, and gastric juice, disseminated tuberculosis was diagnosed. Combination anti-mycobacterial therapy consisting of isoniazid, rifabutin, ethambutol, and pyrazinamide was initiated on day 72. The M. tuberculosis infection was successfully treated, and the lymph nodes shrank on day 195 and were undetectable on day 254 , as confirmed by CT.

\section{Discussion}

It has been reported that recipients of hematopoietic stem cell transplantation (HSCT) as well as solid organ transplantation are highly susceptible to tuberculosis due to long-term immunosuppression. ${ }^{4-6}$ Since the clinical presentation of tuberculosis in transplant recipients is sometimes atypical and diverse, the diagnosis of tuberculosis in this setting is often challenging and difficult, ${ }^{6}$ resulting in both the delayed initiation of anti-mycobacterial therapy and poor prognosis. In addition, HSCT recipients during neutropenia are generally isolated in a positive-pressure room equipped with high-efficiency particulate air filtration, which has a great impact on hospital management if these patients develop tuberculosis. Recently, it has been reported that PET is more sensitive than standard CT at detecting extra-pulmonary lesions (e.g., lymph node, bone, and visceral organs) of tuberculosis, but this was not the case with pulmonary lesions. ${ }^{3}$ Therefore, in our case, the differential diagnosis of the isolated lymph node lesions detected by FDG-PET without significant swelling or pulmo- 
nary shadows should have included tuberculosis, although ATLL is a highly PET-avid disease whose recurrence is often experienced even shortly after successful chemotherapy. If tuberculosis had been optimally diagnosed by lymph node biopsy or interferon-gamma releasing assay before the transplantation, the patient would have received anti-mycobacterial treatment, thus avoiding the development of disseminated disease. It is known that patients with cancer are at high risk for M. tuberculosis infection and that hematological malignancies make patients more susceptible to tuberculosis compared with other cancers. ${ }^{7}$ Some proportions of these patients with hematological malignancies are likely to proceed to HSCT, which is also known to increase the risk of developing $M$. tuberculosis infection. ${ }^{5,6}$ Recently, the usefulness of interferon-gamma releasing assay in HSCT recipients before transplantation in predicting the development of tuberculosis has been demonstrated. ${ }^{8}$ Therefore, interferon-gamma releasing assay should be performed routinely as a pre-transplant examination in regions with a high prevalence of tuberculosis, such as Asian countries. In addition, antimicrobial agents with anti-mycobacterial activities, such as fluoroquinolones, which were indeed administered to our patient, could alter the clinical course of tuberculosis. Physicians should always be aware of this activity when selecting antimicrobial agents.

In conclusion, tuberculosis should be recognized as a possible cause of isolated lymph node lesions identified by FDGPET, and further examination for tuberculosis is essential before proceeding to transplantation.

\section{Acknowledgments}

The authors are indebted to Yuri Niizuma and Kaori Yanai for their skillful data management, and to Satomi Yamanaka for her dedicated care of the patient and her family member.

\section{Conflicts of interest}

The authors declare no conflict of interest.
Author contributions: RA and TM took care of the patient and wrote the manusript; MS, JK, NH, TK, TT, YT and NH took care of the patient and provided important opinions about the manuscript; SO supervised patient care, and reviewed and approved the manuscript.

\section{References}

1. Juweid ME, Cheson BD. Positron-emission tomography and assessment of cancer therapy. N Engl J Med. 2006; 354: 496507.

2. Kaya A, Ergul N, Kaya SY, et al. The management and the diagnosis of fever of unknown origin. Expert Rev Anti Infect Ther. 2013; 11: 805-815.

3. Stelzmueller I, Huber H, Wunn R, et al. 18F-FDG PET/CT in the initial assessment and for follow-up in patients with tuberculosis. Clin Nucl Med. 2015 Dec 22. [Epub ahead of print] DOI: 10.1097/RLU.0000000000001102

4. Russo RL, Dulley FL, Suganuma L, et al. Tuberculosis in hematopoietic stem cell transplant patients: case report and review of the literature. Int J Infect Dis. 2010; 14（Suppl 3): e187-191.

5. Al-Anazi KA, Al-Jasser AM, Alsaleh K. Infections caused by Mycobacterium tuberculosis in recipients of hematopoietic stem cell transplantation. Front Oncol. 2014; 4: 231. doi: 10.3389/fonc.2014.00231. eCollection 2014.

6. Muñoz P, Rodríguez C, Bouza E. Mycobacterium tuberculosis infection in recipients of solid organ transplants. Clin Infect Dis. 2005; 40: 581-587.

7. Kamboj M, Sepkowitz KA. The risk of tuberculosis in patients with cancer. Clin Infect Dis. 2006; 42: 1592-1595.

8. Lee YM, Lee SO, Choi SH, et al. A prospective longitudinal study evaluating the usefulness of the interferon-gamma releasing assay for predicting active tuberculosis in allogeneic hematopoietic stem cell transplant recipients. J Infect. 2014; 69: $165-173$. 